\title{
A Review of Approaches in Steviol Glycosides Synthesis
}

\author{
Nurul Hidayah Samsulrizal ${ }^{1 *}$, Abdul Latif Noh $^{1}$, Tamil CM Sundram ${ }^{1}$, Zarina \\ Zainuddin ${ }^{1}$
}

\begin{abstract}
Stevia rebaudiana (Bertoni) is a commercially important plant worldwide. The leaves of Stevia rebaudiana contain steviol glycosides which are non-caloric, high-potency sweeteners. They are suitable for substituting sucrose and other artificial sweetening agents. Stevia rebaudiana also has many different therapeutic uses, with antidiabetic, anti-cariogenic, antimicrobial, anticancer and antioxidative properties. Rebaudioside A and stevioside are the major glycosides produced in its leaves. However, development of new varieties of Stevia rebaudiana with a greater content of rebaudioside A and decreased content of stevioside is the main concern lately. This is due to rebaudioside A having a more desirable sweet flavour taste than stevioside which possesses bitter aftertaste. In respect to that many biotechnological approaches are being used for the industrial improvement and manipulation of steviol glycosides content of Stevia rebaudiana. Transcriptome profiling has emerged as a useful tool to identify target genes involved in the steviol glycosides biosynthesis pathway. Understanding the mechanism and biosynthesis pathway of these compounds has further helped to improve the glycosides profile by up-regulating and down-regulating the desired genes. The aim of this paper is to describe the latest development in the transcriptome profiling in Stevia rebaudiana as well as to discuss the methods used in this endeavour.
\end{abstract}

\author{
ARTICLE HISTORY \\ Received \\ 13 June 2019 \\ Accepted \\ 05 August 2019
}

\section{KEYWORDS}

Stevia rebaudiana, steviol glycoside, transcriptome

\section{Introduction}

Stevia rebaudiana (Bertoni) which is also known as stevia is a shrubby and herbaceous plant species belonging to the Asteraceae family [30]. It is an indigenous plant of South America which is found to be perennial throughout the year in the highland regions of northeastern Paraguay [1, 31]. S. rebaudiana is the only species out of 230 others, besides Stevia phlebophylla, that is reported to possess the unique ability of accumulating lowcalorie sweetening agents called steviol glycosides (SGs) [2]. Among the different steviol glycosides produced in stevia leaves, stevioside is the most abundant followed by rebaudioside A. These two major glycosides have the potential to become healthier replacement for table sugars as they have zero calories and a desirable taste profile, being 300 times sweeter than sucrose $[3,32]$. The continuously increasing demand for SGs

\footnotetext{
${ }^{1}$ Department of Plant Science, Kulliyyah of Science, International Islamic University Malaysia, Bandar Indera Mahkota, 25200 Kuantan, Pahang, Malaysia

*Corresponding Author: Nurul Hidayah Samsulrizal, e-mail: hidayahsamsulrizal@iium.edu.my
} 
production worldwide as an alternative sugar intensifies the commercial value of this plant in both biopharmaceutical and food and beverages industries [33]. New researches have emerged by adopting various biotechnological strategies and approaches to understand, stimulate or improve the biosynthesis of these secondary metabolites in stevia. In this respect, transcriptome has gained more attention and become promising methods to assess the genetic diversity of the plant's molecular characteristics [34]. As such, transcriptome analysis is widely used to identify gene expressions that are involved in the biosynthesis of steviol glycosides in the leaf tissues and under certain circumstances as well [34]. This review aims to provide a summary on the latest development of the genetic profiles of steviol glycosides and the strategies that are involved in the transcriptomics level.

\section{Stevia rebaudiana}

Stevia rebaudiana (Bertoni) belongs to the family Asteraceae [35], or sunflower family, which is among the largest families of flowering plants, spreading across 1,620 genera and 13 subfamilies [4]. The genus Stevia Cav. is one of the genera within the tribe Eupatorieae which is unique due to its flower morphology [5]. It comprises of approximately 230 species of annual and perennial herbaceous, shrub and sub-shrub plants [1] that live naturally in multiple places including mountain regions, river borders and dry valleys [6]. Out of all these species, S. rebaudiana is the only plant that produces highly valuable sweetening agents with desirable taste profile [2]. It is a short-day plant with a critical day length of approximately 12 to 13 hours [7, 36]. However, the critical day length may vary among cultivars from as early as 8 hours to as long as 14 hours, depending on their photoperiod sensitivity [7, 8 and 9]. Stevia plant morphology includes extensive root system with brittle stems and small, elliptical leaves that occur in alternate arrangement [3, 37]. As described by Ceunen et al. (2012), SGs production mainly occurs in the leaves of the plant in which 33 glycosylated diterpenes of kaurene-type have been discovered whereas a lesser amount is found within its flowers and stem, and almost none in its roots [38]. Apart from the sweetening glycosides, the leaves of stevia also contain essential amino acids, minerals such as phosphorus, potassium, sodium and calcium as well as phytochemicals such as flavonoids, alkaloids, hydroxycynnamic acids and triterpenes [2, 3]. Karimi et al. (2017) reported that the plant's various secondary metabolites have given it a range of therapeutic properties including anti- 
hypercglycaemic, anti-hypertensive, anti-oxidative, anti-microbial, anti-cariogenic and anti-carcinogenic (Table 1). It is thus referred as a sweetening plant with great pharmaceutical significance in comparison to other natural and synthetic sweeteners following its assorted chemical and nutritional constituents and their medicinal attributes [10].

Table 1 Medicinal properties in Stevia rebaudiana compound

\begin{tabular}{ccc}
\hline Compound & Medicinal properties & Reference \\
\hline Stevioside & Anti-inflammantory & {$[11]$} \\
& Anti-hyperglycemic & {$[12]$} \\
& Hypotensive & {$[13]$} \\
Rebaudioside (Rb) A & PTZ-induced convulsions effects & {$[14]$} \\
Dulcoside A & Effect on glycemic & {$[15]$} \\
Steviol & Renal function & {$[16]$} \\
\hline
\end{tabular}

\section{Steviol Glycosides}

Steviol Glycosides (SGs) are well-known secondary metabolites in stevia. They are nonnutritive, non-toxic, high-potency sweeteners with commercially important uses in biopharmaceutical, food and beverages industries [17]. The nine most common diterpenoids that have been identified in the leaf tissues of stevia include stevioside, rebaudiosides $\mathrm{A}$ to $\mathrm{E}$, dulcosides, steviobiosides and rubusosides [18]. Among all these SGs, stevioside and rebaudioside A are classified to be the major sweeteners in stevia [39]. The measure of the sweetness quality of stevia is attributed to the ratio of stevioside and rebaudioside A content in its leaves. Stevioside is described to be most bountiful by accommodating $60-70 \%$ of the total SGs content which is two-fold the amount of rebaudioside A [40]. However, it has a slightly less sweet taste with 300 times the sweetness of sucrose $[1,19]$. They are also reported to be negatively correlated in which higher content of rebaudioside A that will give a more desirable taste profile since stevioside has a lingering effect of pungency and a bitter aftertaste [1]. Accordingly, these sweeteners have a great demand to be utilised as table sugar substitute since the sweetening effect of these compounds is purely taste and they are not metabolised in the human body [1]. In other words, it possesses acceptable sweetening taste at a healthy value. Therefore, it is a great alternative sugar for diabetic patients and those planning to 
control their blood glycemic index since the sweet compounds can pass through the digestive system without chemically breaking down hence producing zero calories $[1,41]$.

\section{Biosynthesis of Steviol Glycosides}

The biosynthesis of SGs partly shares a common route with gibberellins through plastidal methyl erythritol 4-phosphate (MEP) and cytosolic mevalonic acid (MVA) pathways (Fig 1). Both of these metabolites are mainly synthesised in the mesophyll cells of the leaves and almost untraceable in the roots $[1,18,20]$. The initial phase of steviol biosynthesis occurs in plastid which involves the localised MEP pathway where several enzymes consecutively work to catalyse the production of isopentenyl pyrophosphate [42]. These enzymes include deoxyxylulose phosphate synthase (DXS), deoxyxylulose phosphate reductase (DXR), 4-diphosphocytidyl-2-C-methyl-d-erythritol synthase (CMS), 4diphosphocytidyl-2-C-methyl-d-erythritol kinase (CMK), 4-diphosphocytidyl-2-Cmethyl-d-erythritol 2,4-cyclodiphosphate synthase (MCS), 1- hydroxy2-methyl-2(E)butenyl-4-diphosphate synthase (HDS) and 1-hydroxy-2-methyl-2(E)-butenyl-4diphosphate reductase (HDR) [18,20]. 


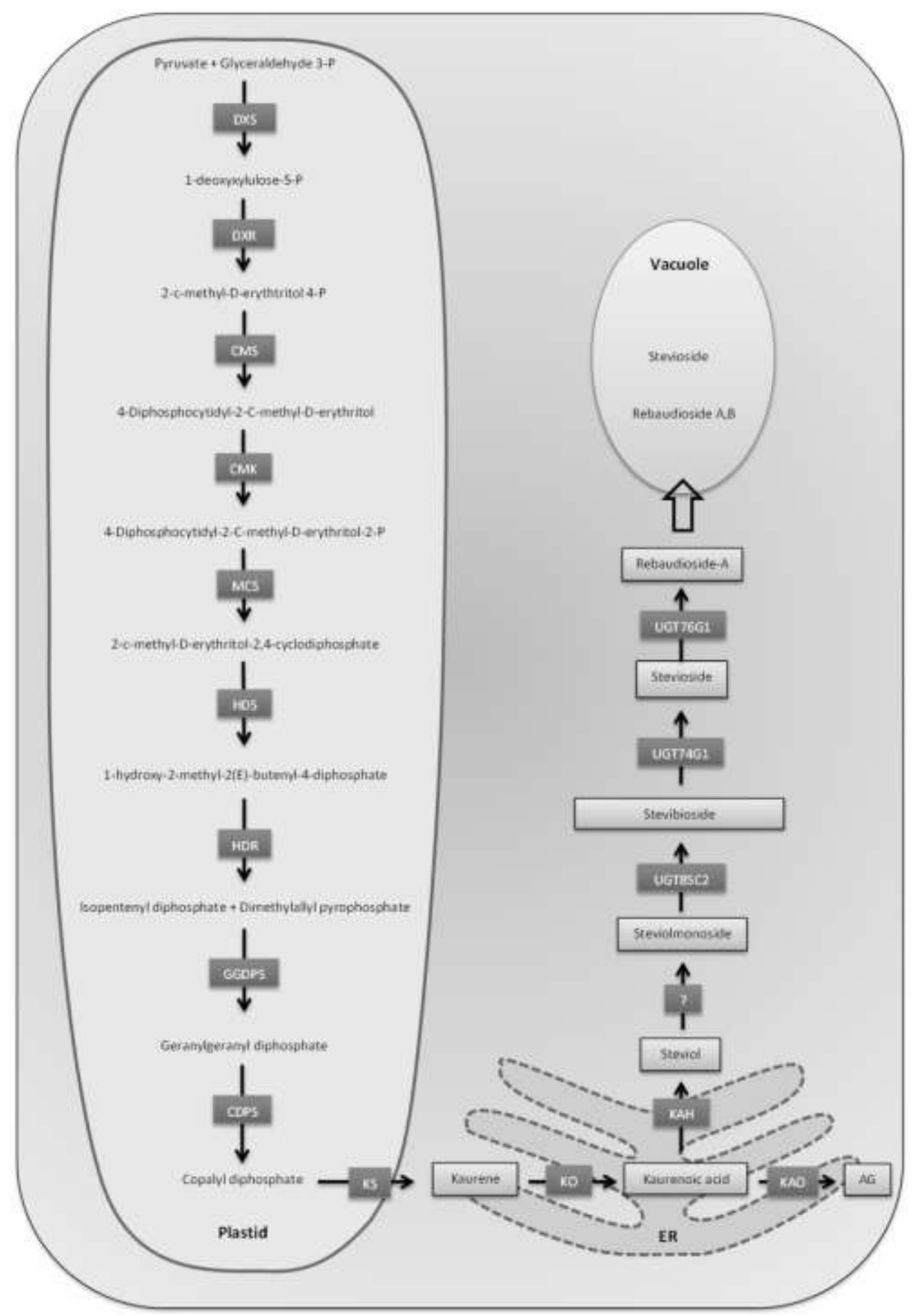

Fig 1 Diagrammatic representation of genes involved in SGs biosynthesis pathway. Abbreviations are as follows: DXS (1-deoxy-D-xylulose-5-phosphate synthase), DXR (1-deoxy-D-xylulose 5phosphate reductoisomerase), CMS (2-C-methyl-D-erythritol 4-phosphate cytidylyltransferase), CMK (4-diphosphocytidyl-2-C-methyl-D-erythritol kinase), MCS (2-C-methyl-D-erythritol 2,4cyclodiphosphate synthase), HDS (4-hydroxy-3-methylbut-2-enyl diphosphate synthase), HDR (4-hydroxy-3-methylbut-2-enyl diphosphate reductase), GGDPS (geranylgeranyl diphosphate synthase), CDPS (ent-copalyldiphosphate synthase), KS (ent-copalyl diphosphate synthase), KO (ent-kaurene oxidase), KAH (ent-kaurenoic acid 13- hydroxylase), UGT85C2 (UDP- 
glycosyltransferase 85C2), UGT74G1 (UDP-glycosyltransferase 74G1),UGT76G1 (UDPglycosyltransferase 76G1), UGT? (unknown UGT), and KAO (ent-kaurenoic acid oxidase).

The second stage of the steviol biosynthesis occurs when geranylgeranyl diphosphate synthase (GGDPS) condenses four isoprene units of isopentenyl pyrophosphate to produce geranylgeranyl diphosphate which is the common precursor for the synthesis of all diterpenoids [43]. This compound is then converted into ent-kaurenoic acid by the following action of enzymes copalyl diphosphate synthase (CDPS), kaurene synthase (KS) and kaurene oxidase (KO) [20]. The steviol glycoside and gibberellin pathways diverge at kaurene where two different endoplasmic reticulum-membrane located cytochrome P450 monooxygenases (CYPs) acted to convert ent-kaurenoic acid into either steviol by kaurenoic acid hydroxylase $(\mathrm{KAH})$ or gibberellic acid by kaurenoic acid oxidase (KAO) [18].

The final phase involves the glycosylation of steviol in cytosol by UDPglycosyltransferases (UGTs) such as UGT85C2, UGT74G1 and UGT76G1 to form various types of steviol glycosides in which they are then vacuolated $[1,18,20]$. Most of the enzymes involved in the biosynthesis of SGs in stevia have been identified. Therefore, in order to increase the production of SGs, current researches should focus on metabolic engineering of these biosynthetic pathways. Silencing 3 major UGT genes through Agrobacterium mediated gene transformation of S. rebaudiana were found to increase SGs production [17].

\section{Biotechnological Approaches for Steviol Glycosides Improvement in Stevia Rebaudiana}

Biotechnological techniques have offered wide opportunities and novel findings in the engineering of SGs biosynthesis pathway in stevia. These include all tools that can assess the transcriptomics, metabolomics and proteomics of the plant. Among these are random amplified polymorphic DNA (RAPD), inter simple sequence repeat (ISSR), amplified fragment length polymorphism (AFLP), simple sequence repeat (SSR), highperformance thin layer chromatography (HPTLC) and next generation sequencing (NGS) technology [21]. These methods are robust, widely applicable, fast, efficient and costeffective [44]. They require only small amounts of template DNA to produce high information output. With evaluation of genetic diversity made possible, the genomic 
resources from the generated data set have been useful to understand the mechanism and pathway of the secondary metabolites. These has been helpful to improve the sweeteners profile in stevia by up-regulating and down-regulating desired genes. For instance, new researches that focus on DNA-based molecular markers have emerged. These researches aim to establish a better understanding of the genetic variability of stevia genotypes up to the point where they can manipulate the ratio of rebaudioside A and stevioside production in the plants leaves [1].

A vast range of molecular marker technologies have also been used to develop functional molecular markers for diversity characterization and genetic improvement of the plant [18]. Apart from that molecular breeding approaches also have been implemented to increase the dry weight of stevia leaves thus increasing the sweetening compounds yields [21]. These biotechnological approaches not only have significant role in the improvement of SGs production in stevia, but can be extended in improving the plant's overall agronomy, biochemistry, evolutionary studies, genome mapping, morphology and physiology as well.

In near future metabolic engineering will be the stepping stone towards biotechnological production of SGs in heterologous host (such as E. coli, S. cerevisiae, cyanobacteria or moss). As initial step towards improving heterologous production terpenoids, $S$. cerevisiae has been successfully rewired to boost the flux via MVA pathway [22]. Meanwhile, E. coli has been successfully constructed to express two S. rebaudiana entkaurene genes encoding ent-copalyl diphosphate synthase (CPPS) and ent-kaurene synthase (KS) enzymes (CPPS-KS module) [45]. In this study, overexpression of three key enzymes of upstream pathway, DXS, IspA and IDI together with expression of genes encoding GGPPS from Rhodobacter spharoides in E. coli strain MG1655 co-expressing the synthetic CPPS-KS module increased the total production of ent-kaurene by 5 folds [23]. These four enzymes (DXS, IDI, IspA and GGPPS) have been widely reported as rate limiting enzymes of pathways of many diterpenoids [24]. Thus, overexpressing these enzymes could possibly lead to increased production of SGs.

\section{Transcriptome Profiling of Genes Related to the Biosynthesis of Steviol Glycosides}

The most significant primary step to optimise the amount of SGs produced in the leaves of stevia is by analysing the genes transcripts that are related to these metabolites' biosynthesis. According to Nature, (2018), the study of an organism's complete set of 
RNA transcripts by using high-throughput modus is called transcriptomics [25]. These transcripts are fabricated either under exclusive conditions or in specialised cells by the organism's genome. Transcriptomics enable the identification of target genes that are useful for further studies in metabolic engineering besides providing a way to understand the genomics of non-model plant without reference genome like stevia. RNA-Seq, for instance, is an effective tool in NGS technology [46]. It is commonly used in researches that need to identify the genes that are expressed during SGs production through an indepth transcript profiling. Its ability to measure transcripts in a precise manner while also being effective for annotation uses, discovery of single nucleotide polymorphisms (SNPs) and de novo assembly has made it a popular method in this area of research. An example of this can be seen in a study done by Chen et al. (2014) in which a thorough profiling of the transcriptome of three stevia genotypes (SR-1, SR-2 and SR-3) was successfully demonstrated using the combination of RNA-Seq and digital gene expression (DGE) [26]. In this study, 80,160 unigenes were annotated and 14,211 of the sequences were characterised into 250 specific metabolic pathways using Kyoto Encyclopaedia of Genes and Genomes (KEGG). The gene sequences of all the enzymes commonly related to the SGs biosynthesis were then analysed in which 143 UGTs unigenes were determined. From there, the expression patterns of eight genes, namely, GGDPS, CPPS, KS, KO, $K A H, U G T 85 C 2, U G T 74 G 1$ and $U G T 76 G 1$, were further evaluated using qRT-PCR which confirmed their involvement in the synthesis pathway [47].

In another study, Kim et al., (2015) took a step further to elucidate the biosynthetic routes and spatial distribution of diterpenoids through the integration of metabolomics and transcriptomics [27]. This study explored the biochemical specialisation of the leaf tissues for diterpenoid production (i.e. diterpenoid glycosides and labdane-type diterpenoids) using metabolite profiling and comparative RNA-Seq transcriptomic analysis of two different tissues, trichromes and leaf without trichromes. It was performed on the basis that plant diterpenoids production and build-up only occur in specialised tissues or specific types of cell. The findings from the differential gene expressions confirmed that SGs only accumulate in leaf cells while other labdane-type diterpenoids are stored in the trichromes. Specific enzyme-encoding genes that were engaged in the initial steps of SGs biosynthesis or the MEP pathway, were identified as 1-deoxy-xylulose 5-phosphate synthases (DXS) genes, 1-deoxy-D-xylulose 5-phosphate reductoisomerase (DXR) 
genes, 1-hydroxy-2-methyl-2-(E)-butenyl4-diphosphatereductase (HDR) genes, 2-Cmethyl-D-erythritol 4-phosphate cytidylyltransferase (MCT) genes, 4-(cytidine 59diphospho)-2-C-methyl-D-erythritol kinase (CMK) genes, 2-C-methyl-D-erythritol 2,4cyclodiphosphate synthase (MDS) genes, and 4-hydroxy-3-methylbut-2-enyl diphosphate synthase (HDS) genes. This method of combining metabolomic and transcriptomic analysis has provided a comprehensive overview on the biosynthetic routes of specialised diterpenoids that are distinct to different parts of stevia leaf tissues[48].

Previous studies identified that SGs production level is high in vegetative phase until flower bud emergence, in which its level is the highest, and is followed by a diminishing amount in flowering phase [12]. Hence in a more recent study, Singh et al. (2017) added valuable information on the biosynthesis pathway of SGs in stevia leaves by unravelling in-depth transcriptional profiles of the genes that were involved during different developmental phase transitions (i.e. leaf tissue in vegetative phase, bud phase and flowering phase) [49]. The fact that SGs partially share a biosynthesis route with gibberellic acids and its accumulation in the leaves is ontogeny-dependent makes studying stevia at transcriptional level more complicated. Hence, this research adopted a global transcriptome sequencing approach to effectively comprehend the influence of these phase transitions towards gene expression during SGs biosynthesis. A total of 41,262 genes were annotated in which de novo assembled transcripts using various NGS platforms successfully detected all 46 genes that were involved in the plastidal MEP and cytosolic MVA pathways. Differential gene expression and quantitative analysis of vital genes such as $D X S, H M G R$ and $K A 13 H$, and gene regulators such as WRKY, MYB, NAC and $T F$ s showed the application of metabolic flux between SGs and gibberellic acids production during the transitions [12]. Furthermore, classification of putative candidates such as cytochrome P450 monooxygenases (CYPs) and UGTs enhanced the genomic resources. The information obtained on these candidates is useful for molecular breeding and genetic engineering efforts in order to enrich SGs content, biomass and yield.

The commercial importance of SGs has resulted in using elicitors as a potential method to induce the production of these compounds in stevia $[28,29,51]$. In a study by Lucho et al., (2018), four stress-related elicitors, namely, methyl jasmonate (MeJa), spermidine (SPD), salicylic acid (SA), and paclobutrazol (PBZ) were introduced to investigate their 
effects towards any changes in SGs' contents and the transcript levels of the corresponding biosynthetic genes. Six elicitor-responsive genes were discovered from SGs biosynthesis pathway i.e. $H D R, G G D P S, C D P S, K S, K O$, and $K A H$. These genes commendably can be regulated at the transcriptional level. In terms of the elicitors, MeJa and SPD were found to give positive effects in up-regulating the transcription of the genes related to SGs biosynthesis. Meanwhile, PBZ treatment was shown to down-regulate the genes that encode kaurenoid enzymes. On the other hand, SA treatment did not influence UGT85C2, UGT74G1, and UGT76G1 transcription though it reduced the level of stevioside produced [50]. Overall, this study has offered new insights into the transcriptional response mechanisms in stevia plants under the effect of these elicitors. However, there is still a lack of information about the transcription factors and key regulators that affect the up-regulation and down-regulation of the genes involved. Therefore, studies that highly integrate transcriptomic, metabolomic, and proteomic studies should be carried out to gain better understanding of the gene regulation.

\section{Conclusion}

Among the many biotechnological approaches that are available, RNA-based study or transcriptomics has emerged as one of the promising methods to stimulate and induce SGs biosynthesis in stevia leaves. The transcriptomic profiling of genes involved in the biosynthesis route of SGs enables target genes to be identified and is useful in metabolic engineering of the plant for improvement of the compounds content, biomass and yield. RNA-Seq is a part of NGS technology that is robust, universal, cost-effective and effective to understand the genomics of non-model plant species like stevia. Information obtained through the use of this tool can enhance the understanding of the plant's genomics and assist in further development of various areas including agronomy, biochemistry, genome mapping and evolutionary studies of stevia. For future prospects, extensive researches involving “omics” technology, i.e. transcriptomic, metabolomic and proteomic studies, can be performed in stevia to understand better the underlying chemical processes especially in the regulation of genes and their conversion into functional products such as SGs metabolites and proteins.

\section{Acknowledgements}

We sincerely thank International Islamic University Malaysia (IIUM) Research Initiative Grant Scheme RIGS17-022-0597) for supporting this work. 


\section{References}

1. Yadav, A. K., Singh, S., Dhyani, D., \& Ahuja, P. S. A review on the improvement of stevia [Stevia rebaudiana (Bertoni)]. Canadian Journal of Plant Science, 2011. 91(1), 1-27.

2. Ijaz, M., Pirzada, A. M., Saqib, M., \& Latif, M. Stevia rebaudiana: An alternative sugar crop in Pakistan-a review. Erling Verl. GmbH Co. KG, 2015. 20(2), 88-96.

3. Singh, S. D., \& Rao, G. P. Stevia: The herbal sugar of 21 st Century. Sugar tech, 2005. 7(1), 1724.

4. Tamokou, J. D. D., Mbaveng, A. T., \& Kuete, V. Antimicrobial activities of African medicinal spices and vegetables. In Medicinal Spices and Vegetables from Africa 2017. (pp. 207-237).

5. King, R. M., \& Robison, H. The Genera of the Eupatorieae (Asteraceae), Monographys in Systematic Botany, The Missouri Botanical Garden, 1987. King, Rm; Robinson, H., Eds

6. Sys, E. A., Marsolais, A. A., \& Brandle, J. 1998. U.S. Patent Application No. 08/652,712.

7. Ceunen, S., Werbrouck, S., \& Geuns, J. M. Stimulation of steviol glycoside accumulation in Stevia rebaudiana by red LED light. Journal of plant physiology, 2012. 169(7), 749-752.

8. Valio, I. F. M., \& Rocha, R. F. Effect of photoperiod and growth regulator on growth and flowering of Stevia rebaudiana Bertoni. Japanese Journal of Crop Science, 1977. 46(2), 243-248.

9. Zaidan, L. B., Dietrich, S. M., \& Felippe, G. M. Effect of photoperiod on flowering and stevioside content in plants of Stevia rebaudiana Bertoni. Japanese Journal of Crop Science, 1980. 49(4), 569-574.

10. Gupta, P. Plant tissue culture of Stevia rebaudiana (Bertoni): A review. Journal of Pharmacognosy and Phytotherapy, 2013. 5(2), 26-33.

11. Yasukawa, K., Kitanaka, S. and Seo, S., Inhibitory effect of stevioside on tumor promotion by 12O-tetradecanoylphorbol-13-acetate in two-stage carcinogenesis in mouse skin. Biological and Pharmaceutical Bulletin, 2002. 25(11), pp.1488-1490.

12. Chatsudthipong, V. and Muanprasat, C., Stevioside and related compounds: therapeutic benefits beyond sweetness. Pharmacology \& therapeutics, 2009. 121(1), pp.41-54.

13. Marcinek, K. and Krejpcio, Z., Stevia rebaudiana Bertoni: health promoting properties and therapeutic applications. Journal für Verbraucherschutz und Lebensmittelsicherheit, 2016.11(1), pp.3-8.

14. Uyanikgil, Y., Cavusoglu, T., Balcıglu, H.A., Gurgul, S., Solmaz, V., Ozlece, H.K., Erten, N. and Erbas, O., Rebaudioside A inhibits pentylenetetrazol-induced convulsions in rats. The Kaohsiung journal of medical sciences, 2016. 32(9), pp.446-451.

15. Shin, D.H., Lee, J.H., Kang, M.S., Kim, T.H., Jeong, S.J., Kim, C.H., Kim, S.S. and Kim, I.J., Glycemic effects of rebaudioside A and erythritol in people with glucose intolerance. Diabetes \& metabolism journal, 2016. 40(4), pp.283-289.

16. Melis, M.S., Rocha, S.T. and Augusto, A., Steviol effect, a glycoside of Stevia rebaudiana, on glucose clearances in rats. Brazilian Journal of Biology, 2009. 69(2), pp.371-374.

17. Yadav, S. K., \& Guleria, P. Steviol glycosides from Stevia: biosynthesis pathway review and their application in foods and medicine. Critical reviews in food science and nutrition, 2012. 52(11), 988-998.

18. Singh, G., Singh, G., Singh, P., Parmar, R., Paul, N., Vashist, R., \& Kumar, S. Molecular dissection of transcriptional reprogramming of steviol glycosides synthesis in leaf tissue during developmental phase transitions in Stevia rebaudiana Bert. Scientific reports, 2017. 7(1), 11835.

19. Modi, A., Litoriya, N., Prajapati, V., Rafalia, R., \& Narayanan, S. Transcriptional profiling of genes involved in steviol glycoside biosynthesis in Stevia rebaudiana bertoni during plant hardening. Developmental Dynamics, 2014. 243(9), 1067-1073.

20. Lucho, S. R., do Amaral, M. N., Milech, C., Ferrer, M. Á., Calderón, A. A., Bianchi, V. J., \& Braga, E. J. B. Elicitor-Induced Transcriptional Changes of Genes of the Steviol Glycoside 
Biosynthesis Pathway in Stevia rebaudiana Bertoni. Journal of Plant Growth Regulation, 2018. 115 .

21. Karimi, R., Vahedi, M., Pourmazaheri, H., \& Balilashaki, K. Biotechnological approaches in Stevia rebaudiana and its therapeutic applications. Advances in Biomedicine and Pharmacy, 2017. 4(1): 31-43.

22. Kampranis, S. C., \& Makris, A. M. Developing a yeast cell factory for the production of terpenoids. Computational and structural biotechnology journal, 2012. 3(4), e201210006.

23. Kong, M. K., Kang, H. J., Kim, J. H., Oh, S. H., \& Lee, P. C. Metabolic engineering of the Stevia rebaudiana ent-kaurene biosynthetic pathway in recombinant Escherichia coli. Journal of biotechnology, 2015. 214, 95-102.

24. Ohto, C., Muramatsu, M., Obata, S., Sakuradani, E., \& Shimizu, S. Prenyl alcohol production by expression of exogenous isopentenyl diphosphate isomerase and farnesyl diphosphate synthase genes in Escherichia coli. Bioscience, biotechnology, and biochemistry, 2009. 73(1), 186-188.

25. Nature. https://www.nature.com/subjects/transcriptomics (accessed on 5 July 2018).

26. Chen, J., Hou, K., Qin, P., Liu, H., Yi, B., Yang, W., \& Wu, W. RNA-Seq for gene identification and transcript profiling of three Stevia rebaudiana genotypes. BMC genomics, 2014.15(1), 571.

27. Kim, M. J., Jin, J., Zheng, J., Wong, L., Chua, N. H., \& Jang, I. C. Comparative transcriptomics unravel biochemical specialization of leaf tissues of Stevia (Stevia rebaudiana) for diterpenoid production. Plant physiology, 2015. pp-01353.

28. Gupta, P., Sharma, S., \& Saxena, S. Effect of salts ( $\mathrm{NaCl}$ and $\mathrm{Na} 2 \mathrm{CO} 3)$ on callus and suspension culture of Stevia rebaudiana for steviol glycoside production. Applied biochemistry and biotechnology, 2014. 172(6), 2894-2906.

29. Yoneda, Y., Nakashima, H., Miyasaka, J., Ohdoi, K., \& Shimizu, H. Impact of blue, red, and farred light treatments on gene expression and steviol glycoside accumulation in Stevia rebaudiana. Phytochemistry, 2017. 137, 57-65.

30. Soejarto, D.D., Botany of Stevia and Stevia rebaudiana. In Stevia 2001. (pp. 31-52). CRC Press.

31. Ramesh, K., Singh, V. and Megeji, N.W. Cultivation of stevia [Stevia rebaudiana (Bert.) Bertoni]: A comprehensive review. Advances in Agronomy, 2006. 89, pp.137-177.

32. Philippe, R.N., De Mey, M., Anderson, J. and Ajikumar, P.K.. Biotechnological production of natural zero-calorie sweeteners. Current Opinion in Biotechnology, 2014.26, pp.155-161.

33. Mthembu, Z. Development of a hairy root bioreactor from Stevia rebaudiana to produce steviol glycosides, 2017. (Doctoral dissertation, Stellenbosch: Stellenbosch University).

34. Brandle, J.E., Richman, A., Swanson, A.K. and Chapman, B.P., Leaf ESTs from Stevia rebaudiana: a resource for gene discovery in diterpene synthesis. Plant molecular biology, 2002. 50(4-5), pp.613-622.

35. Oliveira, V.M.D., Forni-Martins, E.R., Magalhães, P.M. and Alves, M.N. Chromosomal and morphological studies of diploid and polyploid cytotypes of Stevia rebaudiana (Bertoni) Bertoni (Eupatorieae, Asteraceae). Genetics and molecular biology, 2004. 27(2), pp.215-222.

36. Metivier, J. and Viana, A.M. The effect of long and short day length upon the growth of whole plants and the level of soluble proteins, sugars, and stevioside in leaves of Stevia rebaudiana Bert. Journal of Experimental Botany, 1979. 30(6), pp.1211-1222.

37. Abdullateef, R.A. and Osman, M. Studies on effects of pruning on vegetative traits in Stevia rebaudiana Bertoni (Compositae). International Journal of Biology, 2012. 4(1), p.146.

38. Ceunen, S. and Geuns, J.M., 2013. Steviol glycosides: chemical diversity, metabolism, and function. Journal of natural products, 76(6), pp.1201-1228.

39. Mizutani, K. and Tanaka, O. Use of Stevia rebaudiana sweeteners in Japan. In Stevia, 2001. (pp. 191-209). CRC Press.

40. Chiew, M.S., Lai, K.S., Hussein, S. and Abdullah, J.O. A Review on Induced Mutagenesis of Stevia rebaudiana Bertoni. Pertanika Journal of Scholarly Research Reviews, 2016. 2(3). 
41. Shivanna, N., Naika, M., Khanum, F. and Kaul, V.K. Antioxidant, anti-diabetic and renal protective properties of Stevia rebaudiana. Journal of Diabetes and its Complications, 2013. 27(2), pp.103-113.

42. Totté, N., Charon, L., Rohmer, M., Compernolle, F., Baboeuf, I. and Geuns, J.M. Biosynthesis of the diterpenoid steviol, an ent-kaurene derivative from Stevia rebaudiana Bertoni, via the methylerythritol phosphate pathway. Tetrahedron Letters, 2000. 41(33), pp.6407-6410.

43. Kazmi, A., Khan, M.A., Mohammad, S., Ali, A., Kamil, A., Arif, M. and Ali, H., Elicitation directed growth and production of steviol glycosides in the adventitious roots of Stevia rebaudiana Bertoni. Industrial Crops and Products, 2019. 139, p.111530.

44. Abdelkrim, J., Robertson, B.C., Stanton, J.A.L. and Gemmell, N.J., Fast, cost-effective development of species-specific microsatellite markers by genomic sequencing. BioTechniques, 2009. 46(3), pp.185-192.

45. Wang, J., Li, S., Xiong, Z. and Wang, Y., Pathway mining-based integration of critical enzyme parts for de novo biosynthesis of steviolglycosides sweetener in Escherichia coli. Cell research, 2016. 26(2), p.258.

46. Marguerat, S. and Bähler, J. RNA-seq: from technology to biology. Cellular and molecular life sciences, 2010. 67(4), pp.569-579.

47. Gerwig, G.J., te Poele, E.M., Dijkhuizen, L. and Kamerling, J.P. Stevia glycosides: chemical and enzymatic modifications of their carbohydrate moieties to improve the sweet-tasting quality. In Advances in carbohydrate chemistry and biochemistry 2016. (Vol. 73, pp. 1-72). Academic Press.

48. Zheng, J., Zhuang, Y., Mao, H.Z. and Jang, I.C., Overexpression of SrDXS1 and SrKAH enhances steviol glycosides content in transgenic Stevia plants. BMC plant biology, 2019. 19(1), p.1.

49. Nasrullah, N., Ahmad, J., Saifi, M., Rafiqi, U., Quadri, N., Shah, I.G. and Abdin, M.Z., Metabolic profiling and expression analysis of key genes during leaf maturation of Stevia rebaudiana bertoni. Pharmacognosy Magazine, 2018. 14(57), p.327.

50. Lucho, S.R., do Amaral, M.N., Benitez, L.C., Milech, C., Kleinowski, A.M., Bianchi, V.J. and Braga, E.J.B., Validation of reference genes for RT-qPCR studies in Stevia rebaudiana in response to elicitor agents. Physiology and molecular biology of plants, 2018 .24(5), pp.767-779.

51. Vazquez-Hernandez, C., Feregrino-Perez, A.A., Perez-Ramirez, I., Ocampo-Velazquez, R.V., Rico-García, E., Torres-Pacheco, I. and Guevara-Gonzalez, R.G. Controlled elicitation increases steviol glycosides (SGs) content and gene expression-associated to biosynthesis of SGs in Stevia rebaudiana B. cv. Morita II. Industrial Crops and Products, 2019. 139, p.111479. 